\title{
On the state feedback control of inverted pendulum with hysteretic nonlinearity
}

\author{
Mikhail E. Semenov ${ }^{1,2, a}$, Dmitry V. Grachikov ${ }^{2}$, Anton G. Rukavitsyn ${ }^{2}$, and Peter A. Meleshenko ${ }^{2,3}$ \\ 1 Meteorology Department, Zhukovsky-Gagarin Air Force Academy, Starykh Bolshevikov st. 54 “A”, 394064, Voronezh, \\ Russia \\ 2 Digital Technologies Department, Voronezh State University, Universitetskaya sq. 1, 394006, Voronezh, Russia \\ 3 Communication Department, Zhukovsky-Gagarin Air Force Academy, Starykh Bolshevikov st. 54 "A", 394064 , \\ Voronezh, Russia
}

\begin{abstract}
In this paper we consider the mathematical model of the inverted pendulum with the hysteretic nonlinearity (in the form of backlash) under state feedback control. The analytic results for the stability criteria as well as for the solution of the linearized equation are observed and analyzed. The theorems that determine the stabilization of the considered system are also formulated and discussed.
\end{abstract}

\section{Introduction}

The problem of the inverted pendulum is of more than 100 years history [1-3], however it remains relevant even in the present days [4-8]. As is well known the model of the inverted pendulum plays the central role in the control theory $[4,7,9-12]$. Moreover, it is well established benchmark problem that provides many challenging problems to control design. Because of their nonlinear nature pendulums have maintained their usefulness and they are now used to illustrate many of the ideas emerging in the field of nonlinear control [13]. Typical examples are feedback stabilization, variable structure control, passivity based control , back-stepping and forwarding, nonlinear observers, friction compensation, and nonlinear model reduction.

It should also be noted that the model of the inverted pendulum (especially, under various kinds of control of motion of the suspension point) is widely used in the various fields of physics, applied mathematics, engineer sciences, neuroscience, economics and others. In order to make an adequately description of the dynamics of real physical and mechanical systems it is necessary to take into account the effects of hysteretic nature such as "backlash", "stops" etc. [14]. The mathematical models of such nonlinearities according to the classical patterns of Krasnosel'skii and Pokrovskii [15], reduce to operators that are treated as converters in an appropriate function spaces. The dynamics of such converters are described by the relation of "inputstate" and "state-output".

As is known, most of the real physical and technical systems contain a various kind of parts that can be represented as a cylinder with a piston [14]. The backlashes appear in such systems during its long operation due to the "aging" of the materials. As was mentioned above such backlashes are of hysteretic nature and the analysis of such nonlinearities is quiet important and actual problem [16]. In this paper we investigate the problem of the state feed-

\footnotetext{
a e-mail: mkl150@mail.ru
}

back control of the inverted pendulum with the hysteretic nonlinearity in the form of backlash.

\section{Inverted pendulum with hysteretic nonlinearity}

In this section we briefly describe the mathematical model of the inverted pendulum with the horizontal moving suspension point. Also, in terms of the in-out converter we mathematically describe such a nonlinearity as backlash.

\subsection{Mathematical model}

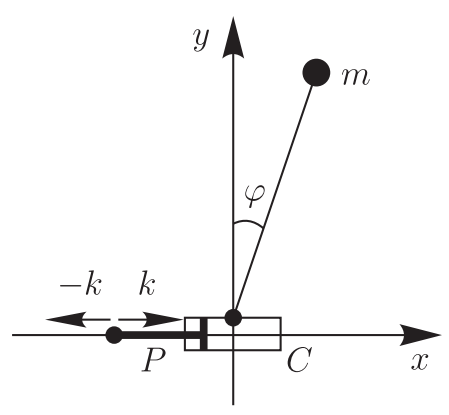

Fig. 1. General view of the inverted pendulum with the suspension point in the form of a cylinder $C$ with a piston $P$.

The equations of motion of the inverted pendulum with the horizontal moving suspension point together with the initial conditions (see the Fig. 1) can be written in the following form:

$$
\begin{gathered}
A \ddot{\varphi}=m g l \sin \varphi-m \ddot{u} l \cos \varphi, \\
\varphi(0)=\varphi_{0}, \dot{\varphi}(0)=\omega(0)=\omega_{0},
\end{gathered}
$$




$$
u(t)=\Gamma\left[u_{0}, h\right] x(t),
$$

where $A$ is a general moment of inertia of the pendulum, $\varphi(t)$ is the angle of vertical deviation of the pendulum, $u(t)$ is a law of motion for the cylinder of the length $h, x(t)$ is a law of motion for the piston which can be interpreted as a control parameter, $\Gamma\left[u_{0}, h\right]$ is defined below.

The equation (1) describes the so-called in-out relations of the hysteretic converter in the form of backlash.

In the following we will consider the case, when the acceleration of the piston is constant, namely

$$
|\ddot{x}|=k=\text { const. }
$$

Let us assume also that the deviations of the pendulum are small so we can rewrite the equation (1) in the linearized form:

$$
\begin{gathered}
A \ddot{\varphi}=m g l \varphi-m \ddot{u} l, \\
\varphi(0)=\varphi_{0}, \dot{\varphi}(0)=\omega(0)=\omega_{0} .
\end{gathered}
$$

\subsection{Backlash as hysteretic nonlinearity}

The mathematical models of the hysteretic nonlinearities according to the classical patterns of Krasnosel'skii and Pokrovskii [15], reduce to operators that are treated as converters in an appropriate function spaces. The dynamics of such converters are described by the relation of "inputstate" and "state-output".

The out state of the converter in the form of backlash (such an out state is considered on the monotonic inputs) can be described by the following relation

$$
u(t)=\Gamma\left[u_{0}, h\right] x(t)=\left\{\begin{aligned}
u_{0}, & \text { for } u_{0} \leqslant x(t) \leqslant u_{0}+h, \\
x(t), & \text { for } x(t)<u_{0}, \\
x(t)-h, & \text { for } u_{0}+h<x(t) .
\end{aligned}\right.
$$

This relation can be illustrated by the Fig. 2 .

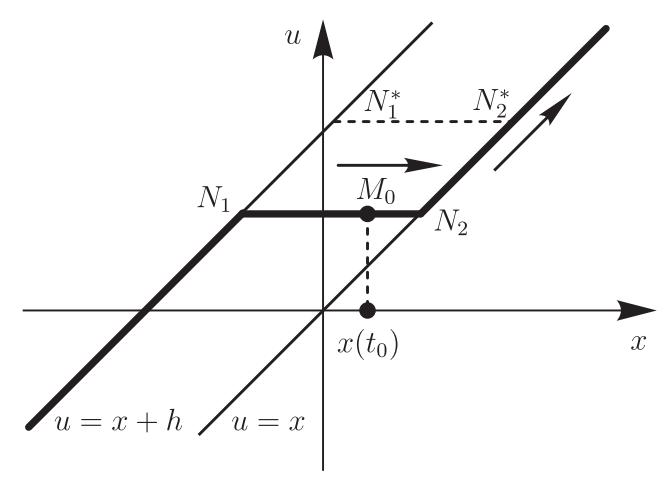

Fig. 2. Schematic view of the backlash (4) action.

With a special limit construction and using the semigroup identity the $\Gamma$-operator can be applied to all piecewise monotonic inputs:

$$
\Gamma\left[u\left(t_{1}\right), h\right] x(t)=\Gamma\left[\Gamma\left[u_{0}, h\right] x\left(t_{1}\right), h\right] x(t) .
$$

It should also be noted that the presence of hysteretic-type operator in the equation (3) complicates the stabilization of the pendulum as a whole. In general, the control impact for such a system will be retarded (we should "predict" the future position of the pendulum).

\section{Stabilization of inverted pendulum with hysteretic nonlinearity}

In this section we consider the state feedback control of the inverted pendulum with hysteretic nonlinearity in the form of backlash. Namely, we obtain the analytic expression for the stability zones of such a system as well as we formulate the theorems that determine the stabilization of the considered system.

\subsection{Dynamical characteristics: analytic results}

Let us consider the state feedback control of the inverted pendulum, i.e., we assume that the input state of the hysteretic converter obeys the following relation:

$$
\ddot{x}=k \operatorname{sign}(\alpha \varphi+\omega),
$$

where $\alpha>0$ and $\operatorname{sign}(z)$ is the usual signum function.

The linearized equation (3) can be rewritten in the equivalent matrix form (we determine the general moment of inertia as $A=m l^{2}$ and use the notation $B=\sqrt{\frac{g}{l}}$ ) as follows:

$$
\left(\begin{array}{c}
\dot{\varphi} \\
\dot{\omega}
\end{array}\right)=V\left(\begin{array}{c}
\varphi \\
\omega
\end{array}\right)+W
$$

where

$$
\begin{gathered}
V=\left(\begin{array}{cc}
0 & 1 \\
B^{2} & 0
\end{array}\right), \quad W=\left(\begin{array}{c}
0 \\
-\frac{\ddot{u}}{l}
\end{array}\right), \\
u(t)=\Gamma\left[u_{0}, h\right] x(t), \ddot{x}=k \operatorname{sign}(\alpha \varphi+\omega), \\
\varphi(0)=\varphi_{0}, \dot{\varphi}(0)=\omega(0)=\omega_{0} .
\end{gathered}
$$

The eigenvalues of the matrix $V$ are $B$ and $-B$ so that the corresponding eigenvectors are $\left(\begin{array}{l}1 \\ B\end{array}\right)$ and $\left(\begin{array}{c}-1 \\ B\end{array}\right)$ respectively. Here, it should be noted that if the phase coordinates of the system under consideration at some time moment will be placed on the line $B \varphi+\omega=0$, then in the future (in the next time moments), in the absence of control, the phase coordinates will asymptotically tend to zero. Therefore, the control should be arranged (on the conceptual level) in such a manner as to "preserve" the phase coordinates in the vicinity of this line.

On each of the interval where the function $\ddot{u}$ is constant the system (7) can be solved and the result is:

$$
\left(\begin{array}{c}
\varphi(t) \\
\omega(t)
\end{array}\right)=\Lambda(t)\left(\begin{array}{c}
\varphi_{0} \\
\omega_{0}
\end{array}\right)+\ddot{u}_{0} v(t)
$$

Here

$$
\begin{gathered}
\Lambda(t)=\left(\begin{array}{cc}
\cosh B t & \frac{1}{B} \sinh B t \\
B \sinh B t & \cosh B t
\end{array}\right) \\
v(t)=\left(\begin{array}{c}
-\frac{1}{g}(\cosh B t-1) \\
-\frac{B}{g} \sinh B t
\end{array}\right),
\end{gathered}
$$

$\varphi_{0}$ and $\omega_{0}$ are initial deviation and frequency of the pendulum respectively, $\ddot{u}_{0}$ is an acceleration of the cylinder on the interval where the function $\ddot{u}$ is constant. 
The behavior of the system (8) on the whole time interval can be represented by the following recurrent relation:

$$
\left(\begin{array}{c}
\varphi_{k}(t) \\
\omega_{k}(t)
\end{array}\right)=\Lambda\left(t-t_{k-1}\right)\left(\begin{array}{c}
\varphi_{t_{k-1}} \\
\omega_{t_{k-1}}
\end{array}\right)+\ddot{u}_{t_{k-1}} v\left(t-t_{k-1}\right) .
$$

Here $t_{k}$ are the moments at which the control changes, $\varphi_{t_{k-1}}$ and $\omega_{t_{k-1}}$ are the values of angle and angle velocity at the moment $t_{k-1}$ respectively, $\ddot{u}_{t_{k-1}}$ is an acceleration of the cylinder on the interval $\left[t_{k-1}, t_{k}\right]$.

\subsection{Dissipative motion}

The equation (3) is called dissipative if there exists a limited domain $\Omega$ on the product of the phase space of the system (7) and the state space of the hysteretic converter (4) that for any initial values $\left(\varphi_{0}, \omega_{0}, u\right) \in \Omega$, the solutions of the equation (3) remain uniformly limited. In other words, this system is called dissipative if there exists a region in the phase space and matching region in the state space of the hysteretic converter that the solution which began in this region do not go to infinity.

Let us formulate the following theorem:

Theorem 1 The sufficient condition for existence of the dissipative regime of the pendulum's motion in a vicinity of the upper position is:

$$
e^{B \tau}\left|B \varphi_{0}+\omega_{0}\right| \leqslant\left|\frac{k B}{g}\right|,
$$

where $\tau=\sqrt{\frac{2 h}{k}}$ is the time for which the piston passes through the cylinder.

Proof As is followed from the equation (10), the movement of the phase parameters on the line $B \varphi+\omega=0$ (such a line corresponds to stabilization of the system) occurs during the time $t$ :

$$
t=\frac{1}{B} \ln \left(\frac{-\frac{B k}{g}}{B \varphi_{0}+\omega_{0}-\frac{B k}{g}}\right)
$$

Let the piston passes through the cylinder during the time $\tau$. Then, the phase parameters of a pendulum are:

$$
B \varphi_{\tau}+\omega_{\tau}=e^{B \tau}\left(B \varphi_{0}+\omega_{0}\right) .
$$

After substitution of (13) in to (12) one gets:

$$
t=\frac{1}{B} \ln \left(\frac{-\frac{B k}{g}}{e^{B \tau}\left(B \varphi_{0}+\omega_{0}\right)-\frac{B k}{g}}\right)
$$

This equation has a real value if

$$
\frac{-\frac{B k}{g}}{e^{B \tau}\left(B \varphi_{0}+\omega_{0}\right)-\frac{B k}{g}}>0
$$

or

$$
e^{B \tau}\left|B \varphi_{0}+\omega_{0}\right|<\left|\frac{B k}{g}\right| .
$$

Let us note that the inequality (11) determines the stability zones of the system under consideration.

\subsection{Non-ideal relay in feedback}

As is known, the measuring devices of any mechanical systems do not always work perfectly. So, let us consider the problem stabilization of the inverted pendulum in the case when the uncertainty in the control takes place. Let us assume that this uncertainty is fixed, then the acceleration of the suspension point (control parameter) corresponds to the output state of the non-ideal relay converter:

$$
\begin{gathered}
y(t)=B \varphi(t)+\omega(t), \\
\ddot{u}=k R\left[-\varepsilon, \varepsilon, \operatorname{sign}\left(\ddot{u}\left(t_{0}\right)\right), y_{0}\right] y(t),
\end{gathered}
$$

where $\varepsilon>0$. Detailed description of this converter is given in [15].

The parameter $\varepsilon$ can be considered as an uncertainty in the measurement of the value $B \varphi+\omega$. Let us assume also that the following inequality takes place:

$$
\varepsilon<\frac{k B}{g} \text {. }
$$

Otherwise the stabilization of the pendulum can not be observed.

Dynamics of the system with non-ideal relay in the feedback described by the equations:

$$
\begin{gathered}
\left(\begin{array}{c}
\dot{\varphi} \\
\dot{\omega}
\end{array}\right)=V\left(\begin{array}{c}
\varphi \\
\omega
\end{array}\right)+W, \\
\ddot{u}=k R\left[-\varepsilon, \varepsilon, \operatorname{sign}\left(\ddot{x}\left(t_{0}\right)\right), y_{0}\right] y(t), \\
\varphi(0)=\varphi_{0}, \omega(0)=\omega_{0}
\end{gathered}
$$

Let us assume that at initial time $y\left(t_{0}\right)=\varepsilon$. The time for which the phase coordinates of the system (9) under influence of the control will move to the position $y\left(t_{c}\right)=$ $-\varepsilon$ can be found using the following expressions:

$$
\begin{gathered}
-\varepsilon=y\left(t_{c}\right) \\
-\varepsilon=\left(\begin{array}{ll}
B & 1
\end{array}\right) \Lambda\left(t_{c}\right)\left(\begin{array}{c}
\varphi_{0} \\
\omega_{0}
\end{array}\right)+\left(\begin{array}{ll}
B & 1
\end{array}\right) k v\left(t_{c}\right) \\
-\varepsilon=e^{B t_{c}} \varepsilon-\frac{k B}{g}\left(e^{B t_{c}}-1\right) \\
t_{c}=\frac{1}{B} \ln \left(\frac{\frac{k B}{g}+\varepsilon}{\frac{k B}{g}-\varepsilon}\right)
\end{gathered}
$$

A similar result takes place if $y\left(t_{0}\right)=-\varepsilon$. Thus, the total period of the control (14) is $T=2 t_{c}$ and $y\left(2 t_{c}\right)=y\left(t_{0}\right)=$ $\varepsilon$. If $y\left(t_{0}\right) \neq \varepsilon$ and $y\left(t_{0}\right) \neq-\varepsilon$, then for a finite time the phase coordinates of the system (under the control (14)) will move to the position $y(t)=\varepsilon$ or $y(t)=-\varepsilon$.

Using the results presented above we can consider the question on the asymptotical (Lyapunov) stability of solutions of the system (15). Namely, we can formulate the following theorem:

Theorem 2 The system (15) has an asymptotically (Lyapunov) stable solution in the form of closed loop:

$\left(\begin{array}{c}\varphi(\theta) \\ \omega(\theta)\end{array}\right)=\left(\begin{array}{cc}\cosh B \theta & \frac{1}{B} \sinh B \theta \\ B \sinh B \theta & \cosh B \theta\end{array}\right)\left(\begin{array}{l}0 \\ \varepsilon\end{array}\right)+\left(\begin{array}{c}-\frac{k}{g}(\cosh B \theta-1) \\ -\frac{B k}{g} \sinh B \theta\end{array}\right)$, 
at $0 \leqslant \theta<t_{c}$,

$\left(\begin{array}{c}\varphi(\theta) \\ \omega(\theta)\end{array}\right)=\left(\begin{array}{cc}\cosh B \theta & \frac{1}{B} \sinh B \theta \\ B \sinh B \theta & \cosh B \theta\end{array}\right)\left(\begin{array}{c}0 \\ -\varepsilon\end{array}\right)+\left(\begin{array}{c}\frac{k}{g}(\cosh B \theta-1) \\ \frac{B k}{g} \sinh B \theta\end{array}\right)$,

at $t_{c} \leqslant \theta \leqslant 2 t_{c}$ with the attraction domain for solution $\left|B \varphi_{0}+\omega_{0}\right| \leqslant\left|\frac{k B}{g}\right|$.

The proof of this theorem can be made using the equations (10) and (17).

As an illustration of this result in the Fig. 3 we present the phase portrait of the system (15). The simulation parameters are: $m=1 \mathrm{~kg}, k=0.2 \mathrm{~m} \cdot \mathrm{sec}^{-2}, g=9.8 \mathrm{~m} \cdot \mathrm{sec}^{-2}$, $l=0.3 \mathrm{~m}, \varepsilon=0.02 \mathrm{sec}^{-1}, \varphi_{0}=-0.01, \omega_{0}=0.0771 \mathrm{sec}^{-1}$.

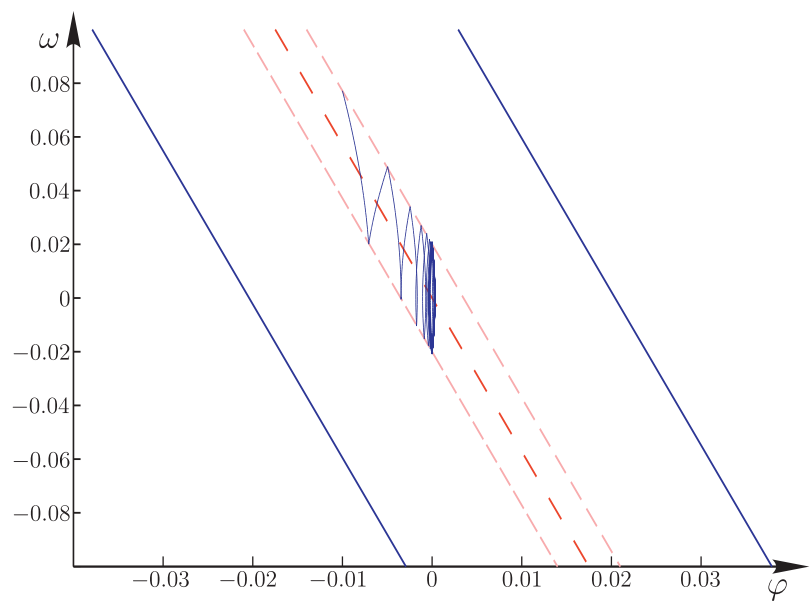

Fig. 3. Phase portrait of the system (15). Blue straight lines limit the zone of dissipative motion; pink lines are $B \varphi+\omega=\varepsilon$ and $B \varphi+\omega=-\varepsilon$; red line is $-B \varphi+\omega=0$. Simulation parameters are $k=0.2 \mathrm{~m} \cdot \mathrm{sec}^{-2}, g=9.8 \mathrm{~m} \cdot \mathrm{sec}^{-2}, l=0.3 \mathrm{~m}, \varepsilon=0.02 \mathrm{sec}^{-1}$, $\varphi_{0}=-0.01, \omega_{0}=0.0771 \mathrm{sec}^{-1}$.

The case when measuring devices have the random uncertainty in the measurements (desynchronization in the control) is of particular interest. In particular, our numerical experiments show that increasing of the simulation time leads to the fact that the probability of the stabilization of the system decreases and tends to zero. This means that the pendulum can not save upright position under desynchronization.

\section{Conclusions}

Here we would like to formulate the following concluding remarks. The obtained in this paper results not only accurately predict the behavior of a pendulum under hysteretic control, but also allow to determine the possibility of the dissipative motion in the vicinity of the top position. The existence of the dissipative motion depends on the initial deviation of the pendulum's position as well as on the physical parameters of the system under consideration. Introduction of non-ideal relay in the state feedback control allows us to describe the periodic modes of the system (7). However, it should also be noted that the results obtained for the presence of the non-ideal relay in the state feedback control can be used for description of the real physical (and, in particular, mechanical) systems because the parameters of such systems can be measured with the inevitable uncertainties only.

Also, our numerical experiments show that the presence of the backlash with the nonzero step in the feedback control of the inverted pendulum leads to dissipative motion only and asymptotic convergence to an upright position is fundamentally unattainable.

\section{References}

1. A. Stephenson, Phil. Mag. 15, (1908) 233

2. P.L. Kapitza, Soviet Phys. JETP 21, (1951) 588

3. P.L. Kapitza, Usp. Fiz. Nauk (in Russian) 44, (1951) 7

4. E.I. Butikov, J. Phys. A: Math. Theor. 35, (2002) 6209

5. A. Arinstein, M. Gitterman, Eur. J. Phys. 29, (2008) 355

6. E.I. Butikov, J. Phys. A: Math. Theor. 44, (2011) 295202

7. J.J. Wang, Simul. Model. Pract. Th. 19, (2011) 440

8. E.I. Butikov, Eur. J. Phys. 33, (2012) 1555

9. F.L. Chernous'ko, S.A. Reshmin, Nonlinear Dynam. 47, (2007) 65

10. S.A. Reshmin, F.L. Chernous'ko, J. Comput. Sys. Sc. Int. 46, (2007) 9

11. K.D. Kim, P. Kumar, IEEE Transactions on Control Systems Technology 21, (2013) 1898

12. J. Huang, et al., IEEE Transactions on Control Systems Technology 21, (2013) 1607

13. K.J. Åström, K. Furuta, Automatica 36, (2000) 287

14. R.A. Nelepin (ed.), Methods of investigation of automatic control nonlinear systems (in Russian) (Nauka, Moscow 1975) 447pp.

15. M.A. Krasnosel'skii, A.V. Pokrovskii, Systems with Hysteresis (Springer Verlag, 1989) 410 pp.

16. M.E. Semenov, D.V. Shevlyakova, P.A. Meleshenko, Nonlinear Dynam. 75, (2014) 247 